\title{
Severe Mitral Insufficiency in Mucopolysaccharidosis Type III-B (Sanfilippo Syndrome)
}

\author{
Joseph Muenzer, ${ }^{1}$ Robert H. Beekman, ${ }^{2}$ Lisa M. Profera, ${ }^{3}$ and Edward L. Bove ${ }^{4}$ \\ Divisions of ${ }^{1}$ Biochemical Genetics and Metabolism, and ${ }^{2}$ Cardiology, ${ }^{3}$ Department of Pediatrics, ${ }^{4}$ Section of \\ Thoracic Surgery, Department of Surgery, University of Michigan Medical School, Ann Arbor, Michigan, USA
}

SUMMARY. A 6-year-old girl with mucopolysaccharidosis (MPS) III-B (Sanfilippo syndrome) who developed severe mitral regurgitation and congestive heart failure requiring surgery (valvuloplasty) is reported. One year after surgery the patient remains well, with marked improvement in her physical activity, and without signs of heart failure. This is only the second report of severe mitral regurgitation in MPS III, and is the first report of a successful repair (valvuloplasty) of a dysplastic mitral valve in the MPS. Mitral valvuloplasty should be considered instead of valve replacement in any MPS patient with mitral valve regurgitation requiring surgery.

\section{KEY WORDS: Mucopolysaccharidoses - Sanfilippo syndrome - Mitral regurgitation - Congestive heart failure - Valvuloplasty}

The mucopolysaccharidoses (MPS) are a heterogeneous group of lysosomal storage disorders characterized by excessive urinary excretion of mucopolysaccharides, progressive mental and physical deterioration, and premature death in the severe forms [3]. Cardiovascular involvement commonly occurs in MPS, and cardiorespiratory failure is a major cause of the early death in MPS I [1, 4]. Severe cardiac involvement has not been reported in MPS III (Sanfilippo syndrome) except for one patient (the diagnosis of MPS III was based on clinical grounds before confirmation by enzymatic analysis was available) with severe mitral valve involvement requiring valve replacement [2]. Mild cardiac involvement has been reported to occur occasionally in MPS III, with thickening of the posterior mitral leaflet and mild asymmetric septal hypertrophy [1].

We describe a patient with MPS III-B (diagnosis confirmed by enzymatic analysis), who developed congestive heart failure requiring surgery for severe mitral regurgitation. Furthermore, the mitral valve was successfully repaired (valvuloplasty), avoiding the risks attendant with mitral valve re-

Address offprint requests to: Dr. Joseph Muenzer, Department of Pediatrics, MSRB II 3570B Box 0688, University of Michigan Medical Center, Ann Arbor, MI 48109, USA. placement and chronic anticoagulation in childhood. Although cardiac disease is common in most patients with MPS, this is only the second report of severe cardiac involvement and its successful treatment in a patient with MPS III.

\section{Case Report}

The patient was born to a 25-year-old woman, gravida 2, para 1, abortus 0 , after an uncomplicated pregnancy at 36 weeks gestation, with a birth weight of $2.41 \mathrm{~kg}$. At age 2 she was noted to have language delay, and a Bailey Scale of infant development found a mental age of 18 months. Physical examination was normal at age 2 years, except for a cardiac murmur and coarse facial features. A diagnosis of MPS III-B was made at approximately 3 years of age, when she developed hepatomegaly, progressive mental deterioration, and coarsening facial features. Alpha- $N$-acetylglucosaminidase activity was not detectable in serum and white blood cells. Normal activity of beta-galactosidase and beta-glucuronidase was present in white blood cells. At age 5.5 years, her development was significantly delayed and she exhibited extreme hyperactivity with aggressive behavior.

A grade II/VI apical systolic regurgitant murmur was noted at 4.5 years of age. There were no symptoms of congestive heart failure, and her weight was at the 75 th percentile. An electrocardiogram demonstrated left ventricular hypertrophy with $T$ wave changes. Chest X-ray showed normal heart size, and a two-dimensional echocardiogram and Doppler study showed moderate mitral regurgitation with a dilated left atrium and left ventricle. The mitral valve was severely prolapsed with a myxomatous 

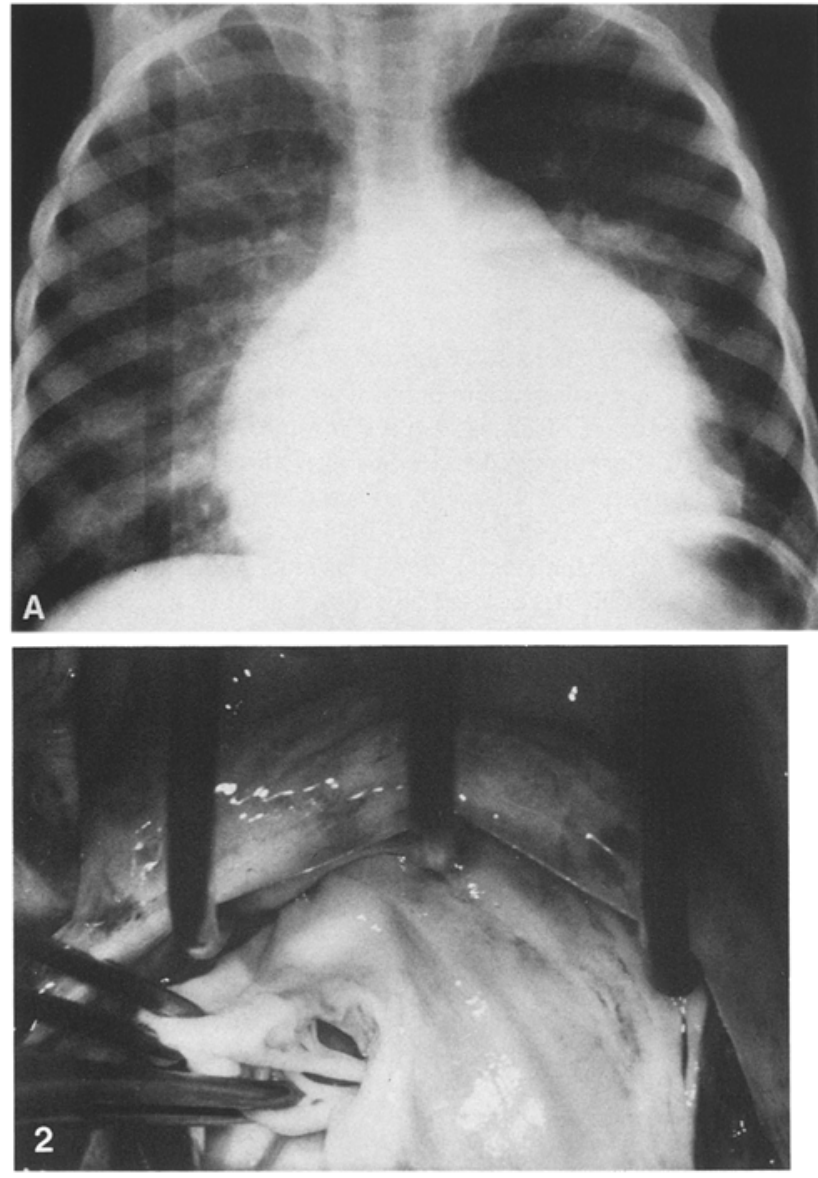

appearance and redundant tissue. The left ventricular shortening fraction was $38 \%$ and the left ventricular ejection fraction was $68 \%$.

At 5.5 years of age she was hospitalized with a 1-month history of congestive heart failure. She was begun on digoxin and diuretic therapy without significant symptomatic improvement. Chest $\mathrm{x}$-ray showed severe cardiomegaly (Fig. 1A). Cardiac catheterization documented severe mitral regurgitation with massive left atrial enlargement and pulmonary artery hypertension. Cardiac output was low at $1.9 \mathrm{~L} / \mathrm{min} / \mathrm{m}^{2}$ and left ventricular end-diastolic pressure was elevated to $26 \mathrm{mmHg}$. The left ventricular diastolic volume was $77 \mathrm{ml} / \mathrm{m}^{2}$, the ejection fraction was $47 \%$, and the mitral regurgitant fraction was $58 \%$ (i.e., severe mitral regurgitation).

At 6 years of age, because of intractable congestive heart failure (weight $<5$ th percentile), mitral valve repair was performed. At surgery, marked prolapse of the anterior leaflet of the mitral valve was found (Fig. 2) and mitral valvuloplasty was performed consisting of shortening of multiple anterior leaflet chordae tendineae into the papillary muscles. Plegetted mattress sutures were placed on both commissures to reduce annular dilatation. Excellent coaptation of the anterior and posterior leaflets was obtained and mitral valve replacement was not necessary. No valve tissue was removed. The patient weaned from cardiopulmonary bypass easily, and had an uneventful postoperative course.

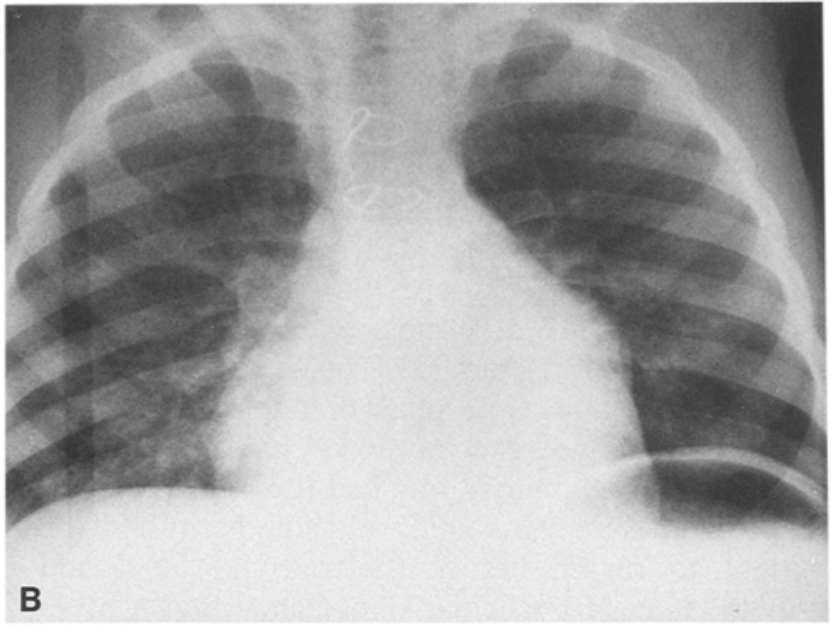

Fig. 1. (A) Preoperative chest $x$-ray in a 6-year-old patient with MPS III-B (Sanfilippo syndrome) demonstrating severe cardiomegaly. (B) A significant decrease in cardiac size was noted at 8 months after mitral valvuloplasty.

Fig. 2. Intraoperative photograph of the mitral valve viewed from the left atrium: anterior and posterior leaflets were thickened with marked prolapse of the anterior leaflet. Forceps shown retracting anterior leaflet. Orientation of photograph: top is anterior and left is superior.

\section{Discussion}

Sanfilippo syndrome (MPS III) is characterized by severe central nervous system involvement, but usually only mild somatic disease. This report documents only the second child with Sanfilippo syndrome (MPS III-B) with severe cardiac involvement reported in the literature. Mild mitral regurgitation was first detected at age 2 years, but over the subsequent 3 years it progressed to severe regurgitation and intractable heart failure with pulmonary artery hypertension and failure to thrive. Although mild One year after surgery, the patient continues to do well,
with marked improvement in her activity level and without symptoms of heart failure. Physical exam reveals only a I/VI systolic regurgitant murmur without a diastolic rumble. The heart size on chest $\mathrm{x}$-ray has improved considerably (Fig. lB). diogram yeal mild residual mitral regurgitation, no mitral stenosis, and a slightly dyskinetic left ventricle. 
cardiac abnormalities have been reported to occur in Sanfilippo syndrome, many patients with MPS III do not have routine cardiac evaluations due to their mental retardation and severe hyperactivity with aggressive behavior.

Cardiac valve replacement has been reported in MPS I H/S, MPS I S, and MPS VI, although experience is limited [4]. The previous reported MPS III patient underwent valve replacement because of severe mitral insufficiency [2]. This is the first report to document successful repair of a dysplastic mitral valve in mucopolysaccharidoses. Mitral valvuloplasty and annuloplasty reduced the mitral regurgitation from severe to mild, and has permitted the patient to regain her normal activity level and growth. It is of particular importance that a prosthetic mitral valve replacement was not necessary in the repair. Prosthetic valves may require replacement with somatic growth, and mechanical valves demand chronic anticoagulation therapy which is difficult in childhood, particularly in children with hyperactivity and psychomotor retardation. Mitral valvuloplasty should be considered instead of valve replacement in any MPS patient with mitral valve dysfunction requiring surgery.

\section{References}

1. Gross DM, Williams JC, Caprioli C, Dominguez B, Howell R (1988) Echocardiographic abnormalities in the mucopolysaccharide storage diseases. Am J Cardiol 61:170-176

2. Herd JK, Subramanian S, Robinson H (1973) Type III mucopolysaccharidosis: Report of a case with severe mitral valve involvement. $J$ Pediatr 82:101-104

3. Neufeld EF, Muenzer J (1989) The mucopolysaccharidoses. In: Scriver CR, Beaudet AL, Sly WS, Valle D (eds) The metabolic basis of inherited disease, 6th ed. McGraw-Hill, New York, pp 1565-1587

4. Pyeritz RE (1983) Cardiovascular manifestations of heritable disorders of connective tissue. In: Steinberg AG, Bearn AG, Molutsky AG, Childs B (eds) Progress in medical genetics, new series, vol 5. WB Saunders, Philadelphia, pp 191-302 